\title{
Solvent control of charge transfer excited state relaxation pathways in $[\mathrm{Fe}(2,2$ '- bipyridine)(CN)4]2-
}

Kjær, Kasper Skov; Kunnus, Kristjan; Harlang, Tobias C. B.; Van Driel, Tim B.; Ledbetter, Kathryn; Hartsock, Robert W.; Reinhard, Marco E.; Koroidov, Sergey; Li, Lin; Laursen, Mads G.

Total number of authors:

37

Published in:

Physical Chemistry Chemical Physics

Link to article, DOI:

10.1039/c7cp07838b

Publication date:

2018

Document Version

Peer reviewed version

Link back to DTU Orbit

Citation (APA):

Kjær, K. S., Kunnus, K., Harlang, T. C. B., Van Driel, T. B., Ledbetter, K., Hartsock, R. W., Reinhard, M. E., Koroidov, S., Li, L., Laursen, M. G., Biasin, E., Hansen, F. B., Vester, P., Christensen, M., Haldrup, K., Nielsen, M. M., Chabera, P., Liu, Y., Tatsuno, H., ... Gaffney, K. J. (2018). Solvent control of charge transfer excited state relaxation pathways in $\left[\mathrm{Fe}\left(2,2^{\prime} \text {-bipyridine }\right)(\mathrm{CN})_{4}\right]^{2}$. Physical Chemistry Chemical Physics, 20(6), 4238-4249. https://doi.org/10.1039/c7cp07838b

\section{General rights}

Copyright and moral rights for the publications made accessible in the public portal are retained by the authors and/or other copyright owners and it is a condition of accessing publications that users recognise and abide by the legal requirements associated with these rights.

- Users may download and print one copy of any publication from the public portal for the purpose of private study or research.

- You may not further distribute the material or use it for any profit-making activity or commercial gain

- You may freely distribute the URL identifying the publication in the public portal 


\title{
Solvent control of charge transfer excited state relaxation pathways in $\left[\mathrm{Fe}\left(2,2^{\prime} \text {-bipyridine }\right)(\mathrm{CN})_{4}\right]^{2-} \dagger$
}

\author{
Kasper S. Kjær, $\quad$ *abc Kristjan Kunnus, ${ }^{a}$ Tobias C. B. Harlang, ${ }^{\text {bc }}$ Tim B. Van Driel, ${ }^{d}$ \\ Kathryn Ledbetter, ${ }^{a}$ Robert W. Hartsock, ${ }^{a}$ Marco E. Reinhard, ${ }^{a}$ Sergey Koroidov, ${ }^{a}$ \\ Lin Li, ${ }^{a}$ Mads G. Laursen, ${ }^{b}$ Elisa Biasin, ${ }^{a b}$ Frederik B. Hansen, ${ }^{b}$ Peter Vester, ${ }^{b}$ \\ Morten Christensen, ${ }^{\mathrm{b}}$ Kristoffer Haldrup, ${ }^{\mathrm{b}}$ Martin M. Nielsen, ' ${ }^{\mathrm{b}}$ Pavel Chabera, ${ }^{\mathrm{c}}$ \\ Yizhu Liu, ${ }^{\text {ce }}$ Hideyuki Tatsuno, ${ }^{c}$ Cornelia Timm, ${ }^{c}$ Jens Uhlig, ${ }^{c}$ Villy Sundstöm, \\ Zoltán Németh, ${ }^{\dagger}$ Dorottya Sárosiné Szemes, ' 'Éva Bajnóczi, ${ }^{\dagger}$ György Vankó, \\ Roberto Alonso-Mori, ${ }^{d}$ James M. Glownia, ${ }^{d}$ Silke Nelson, ${ }^{d}$ Marcin Sikorski, ${ }^{d}$ \\ Dimosthenis Sokaras, ${ }^{9}$ Henrik T. Lemke, ${ }^{\text {dh }}$ Sophie E. Canton, ${ }^{i j}$ Kenneth Wärnmark, ${ }^{e}$ \\ Petter Persson, $\quad{ }^{k}$ Amy A. Cordones ${ }^{a}$ and Kelly J. Gaffney ${ }^{\star a g}$
}

The excited state dynamics of solvated $\left[\mathrm{Fe}(\mathrm{bpy})(\mathrm{CN})_{4}\right]^{2-}$, where bpy $=2,2^{\prime}$-bipyridine, show significant sensitivity to the solvent Lewis acidity. Using a combination of optical absorption and X-ray emission transient spectroscopies, we have previously shown that the metal to ligand charge transfer $(\mathrm{MLCT})$ excited state of $\left[\mathrm{Fe}(\mathrm{bpy})(\mathrm{CN})_{4}\right]^{2-}$ has a 19 picosecond lifetime and no discernable contribution from metal centered (MC) states in weak Lewis acid solvents, such as dimethyl sulfoxide and acetonitrile. ${ }^{1,2}$ In the present work, we use the same combination of spectroscopic techniques to measure the MLCT excited state relaxation dynamics of $\left[\mathrm{Fe}(\mathrm{bpy})(\mathrm{CN})_{4}\right]^{2-}$ in water, a strong Lewis acid solvent. The chargetransfer excited state is now found to decay in less than 100 femtoseconds, forming a quasi-stable metal centered excited state with a 13 picosecond lifetime. We find that this $M C$ excited state has triplet ( $\left.{ }^{3} M C\right)$ character, unlike other reported six-coordinate Fe( $\left.॥\right)$-centered coordination compounds, which form $\mathrm{MC}$ quintet $\left({ }^{5} \mathrm{MC}\right)$ states. The solvent dependent changes in excited state non-radiative relaxation for [Fe(bpy) $\left.(\mathrm{CN})_{4}\right]^{2-}$ allows us to infer the influence of the solvent on the electronic structure of the complex. Furthermore, the robust characterization of the dynamics and optical spectral signatures of the isolated ${ }^{3} \mathrm{MC}$ intermediate provides a strong foundation for identifying ${ }^{3} \mathrm{MC}$ intermediates in the electronic excited state relaxation mechanisms of similar Fe-centered systems being developed for solar applications.

\section{Introduction}

Inorganic complexes are attractive candidates for solar energy applications due to their tunable electronic properties, which can be varied synthetically by changing the metal atom and ligand composition or structure. Molecular photosensitizers based on $4 \mathrm{~d}$ and $5 \mathrm{~d}$ transition metals, such as Ru and Ir, have been successfully implemented in photovoltaic applications

\footnotetext{
${ }^{a}$ PULSE Institute, SLAC National Accelerator Laboratory, Stanford University, Menlo Park, California 94025, USA. E-mail: kaspersk@gmail.com, kgaffney@slac.stanford.edu

${ }^{b}$ Molecular Movies, Department of Physics, Technical University of Denmark, DK-2800, Lyngby, Denmark

${ }^{c}$ Department of Chemical Physics, Lund University, P.O. Box 12 4, 22100 Lund, Sweden

${ }^{d}$ LCLS, SLAC National Accelerator Laboratory, Menlo Park, California 94025, USA

${ }^{e}$ Centre for Analysis and Synthesis, Department of Chemistry, Lund University, P.O. Box 124, 22100 Lund, Sweden

${ }^{f}$ Wigner Research Centre for Physics, Hungarian Academy of Sciences, P.O. Box 49, H-1525 Budapest, Hungary

${ }^{g}$ SSRL, SLAC National Accelerator Laboratory, Menlo Park, California, 94025, USA

${ }^{h}$ SwissFEL, Paul Scherrer Institut, Villigen PSI 5232, Switzerland

${ }^{i}$ ELI-ALPS, ELI-HU Non-Profit Ltd., Dugonics ter 13, Szeged 6720, Hungary

${ }^{j}$ FS-ATTO, Deutsches Elektronen-Synchrotron (DESY), Notkestrasse 85, D-22607 Hamburg, Germany

${ }^{k}$ Theoretical Chemistry Division, Lund University, P.O. Box 124, 22100 Lund, Sweden

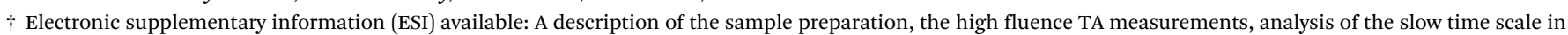
the XES ground state recovery, timing diagnostics, data treatment and computational chemistry. See DOI: 10.1039/c7cp07838b
} 
due to their intense absorption, long-lived charge transfer excited states, and their ability to undergo reversible redox processes. ${ }^{3}$ However, their cost and scarcity render them impractical for large-scale development.

Transferring the functionality of $4 \mathrm{~d}$ and $5 \mathrm{~d}$ transition metal complexes to the more abundant $3 \mathrm{~d}$ transition metal centered systems motivates wide-ranging investigations targeting solar energy applications. ${ }^{4}$ Solar energy applications benefit from long-lived metal-to-ligand charge-transfer (MLCT) excited states, but the majority of optically generated MLCT excited states in 3d transition metal complexes relax on the sub-ps timescale. A central challenge to extending the charge transfer excited state lifetimes in light harvesting complexes based on $3 \mathrm{~d}$ transition metals is the weaker ligand field splitting of $3 \mathrm{~d}$ complexes, relative to their $4 \mathrm{~d}$ or $5 \mathrm{~d}$ counterparts. The smaller ligand field splitting leads to ligand field excited states with lower energies than the optically bright charge transfer excited states that very efficiently quench the MLCT excited states. This deactivation reduces the MLCT lifetime of $3 \mathrm{~d}$ transition metal complexes by orders of magnitude compared to their $4 \mathrm{~d}$ and 5d analogs. For instance, ruthenium(II)-centered polypyridyl compounds are characterized by MLCT states with hundreds of nanoseconds to microseconds lifetimes, ${ }^{5-9}$ the MLCT state of similar complexes with an isoelectronic iron(II) center undergoes spin crossover to a metal centered quintet $\left({ }^{5} \mathrm{MC}\right)$ state within hundreds of femtoseconds. ${ }^{10-24}$

Significant progress in the design of iron-centered molecular systems with extended MLCT lifetimes targeted at solar energy applications has been made recently and demonstrates the potential of suppressing internal conversion and intersystem crossing. ${ }^{2,25-30}$ The synthetic strategy pursued with the most success for octahedral iron(II) complexes has been ligand selection for increased ligand-to-metal $\sigma$-donation, beyond that of typical polypyridyl ligands. Increased $\sigma$-donation leads to larger ligand field splitting and destabilization of the MC excited states relative to MLCT excited states. A range of strong $\sigma$ donating $\mathrm{N}$-heterocyclic carbene ligands have provided Fe-complexes with MLCT lifetimes ranging from $\sim 300$ fs to $\sim 30 \mathrm{ps},{ }^{2,25,27,30,31}$ long enough to allow transfer of the ligandlocalized electron for photovoltaic applications. ${ }^{27,28}$

The metal centered triplet $\left({ }^{3} \mathrm{MC}\right)$ excited state has recently been suggested to play a critical role in mediating the MLCT decay pathway in the cases of both $\mathrm{Fe}(\mathrm{II})$-centered spin crossover complexes (short-lived, $\sim 100 \mathrm{fs}$, MLCT states) ${ }^{21,22}$ and MLCT complexes (long-lived, $\sim 20$ ps, MLCT states), ${ }^{2,26,29,30}$ and has additionally been observed as a precursor for ligand dissociation following excitation of $\left[\mathrm{Fe}(\mathrm{CN})_{6}\right]{ }^{4-32}$ Although still under debate, ${ }^{18}$ much experimental and computational evidence supports the presence of a very short lived ${ }^{3} \mathrm{MC}$ transient state in the spin state transition dynamics to the relatively long lived ${ }^{5} \mathrm{MC}$ state. ${ }^{20-22,33,34}$ In the cases where MLCT lifetimes are extended to the picosecond time scale, they decay without well characterized intermediates, thus preventing experimental identification of decay-mediating states. Quantum chemical and dynamics calculations for several such complexes do suggest that the MLCT lifetime is strongly influenced by the relative positions of the potential curves of the MLCT and ${ }^{3} \mathrm{MC}$ excited states. ${ }^{2,26,29,35-38}$ Although the important role of the ${ }^{3} \mathrm{MC}$ state in facilitating the excited state decay of hexacoordinated Fe(II) complexes has been suggested, a metastable ${ }^{3} \mathrm{MC}$ state has yet to be cleanly isolated for these complexes. Therefore, it is difficult to assess the capacity of experimental methods to identify the role of ${ }^{3} \mathrm{MC}$ intermediates in the excited state dynamics of Fe-based systems.

A strong solvatochromic effect has been well-established for Fe- and Ru-centered cyano-polypyridyl complexes, ${ }^{39-44}$ and has been ascribed to the interaction of high Lewis acidity solvents (such as water) with the $\mathrm{N}$ lone pair of the $\mathrm{CN}^{-}$ligand. Here, we exploit the solvent-dependent electronic structure of $\left[\mathrm{Fe}(\mathrm{bpy})(\mathrm{CN})_{4}\right]^{2-}$, illustrated in Fig. 1, to vary the relative energies of the MLCT and MC states with the intent of generating a metastable ${ }^{3} \mathrm{MC}$. The choice of $\left[\mathrm{Fe}(\mathrm{bpy})(\mathrm{CN})_{4}\right]^{2-}$ was motivated by previous transient optical measurements that demonstrate the loss of characteristic MLCT features from the excited state spectrum upon changing the solvent Lewis acidity. ${ }^{45}$ This study clearly demonstrated the solvent dependence of the non-radiative relaxation in this complex, but did not have the time resolution to determine relaxation rates nor the ability to determine the MC excited state intermediate formed in water. ${ }^{45}$ The use of such a chemically amenable system allows us to develop our current understanding of how metal-ligand bonding controls the internal conversion and intersystem crossing pathways in $3 \mathrm{~d}$ transition metal complexes. In this regard, the solvatochromic $\left[\mathrm{Fe}(\mathrm{bpy})(\mathrm{CN})_{4}\right]^{2-}$ mixed ligand complex provides an excellent model for addressing these fundamental questions in physical chemistry, as the degree of metal-ligand $\sigma$ - and $\pi$-bonding interactions can be varied with the solvent. Steadystate measurements have shown this interaction to shift electron density on the cyanide ligand and increase the metal-to-ligand $\pi$

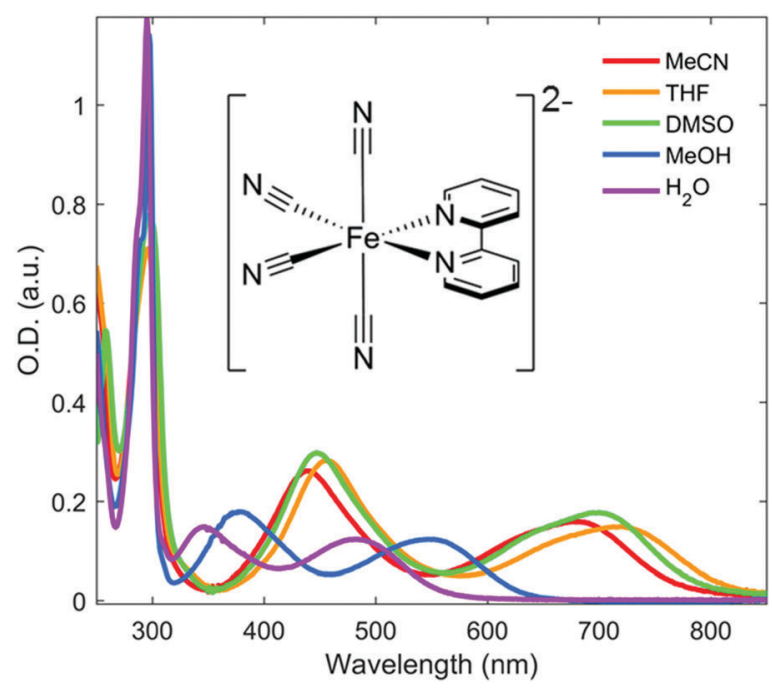

Fig. 1 Solvatochromism of $\left[\mathrm{Fe}(\mathrm{bpy})(\mathrm{CN})_{4}\right]^{2-}$. The inset shows the $\left[\mathrm{Fe}(\mathrm{bpy})(\mathrm{CN})_{4}\right]^{2-}$ system, and the rest of the panel shows its optical absorption spectrum in a series of solvents with varying Lewis acidity; acetonitrile $(\mathrm{MeCN})$ tetrahydrofuran (THF), dimethyl sulfoxide (DMSO), methanol $(\mathrm{MeOH})$, and water $\left(\mathrm{H}_{2} \mathrm{O}\right)$. 
back-donation for the water-solvated complex..$^{39,40,46}$ The highest occupied molecular orbitals (HOMO) of mixed Fe $d\left(t_{2 g}\right)$ and cyano $\pi^{*}$ character are therefore stabilized relative to the lowest unoccupied molecular orbitals (LUMO) of bpy-ligand $\pi^{*}$ character, resulting in a net destabilization of the respective MLCT excited state. ${ }^{47}$ Experimental investigation of the solvent influence on the MC levels is significantly more scarce, but one study finds the trend of solvent-dependent excited state lifetimes for $\mathrm{Ru}$ cyanopolypyridyl complexes can be qualitatively reproduced under the assumption that the MC energy levels are unchanged relative to the ground state. ${ }^{44}$

To characterize the excited state dynamics of $\left[\mathrm{Fe}(\mathrm{bpy})(\mathrm{CN})_{4}\right]^{2-}$ in water we have combined UV-visible pump-probe spectroscopy measuring the transient optical absorption (TA) with transient K-edge X-ray emission spectroscopy (XES), a combination which have delivered key insights in the dynamics of $3 \mathrm{~d}$ transition metal centered systems before. ${ }^{1,21,48-52}$ The signal recorded by TA arises from dipole-allowed transitions in the optical regime, rendering it sensitive to charge transfer excited states involving ligand-localized excited electrons. XES monitors the fluorescence emitted during the 2 p-to-1s or 3 p-to-1s decay following 1 s core-hole ionization of the $\mathrm{Fe}$ metal center. The fluorescence intensity and spectral features are sensitive to the total spin moment on the iron center, and therefore, this method can distinguish MC excited states differing in Fe spin moments, such as ${ }^{3} \mathrm{MC}$ and ${ }^{5} \mathrm{MC}$ states. The combination of the two techniques enables full characterization of the excited state relaxation pathway of $3 d$ transition metal complexes. The present study has unambiguously identified a metastable ${ }^{3} \mathrm{MC}$ excited state with the transient XES spectrum. This identification, in combination with the TA measurements enables the assignment of transient absorption features in the UV-visible spectrum to the ${ }^{3} \mathrm{MC}$ excited state that should prove valuable to the interpretation of electronic excited state relaxation dynamics in other Fe(II) systems.

We report the excited state dynamics of $\left[\mathrm{Fe}(\mathrm{bpy})(\mathrm{CN})_{4}\right]^{2-}$ solvated in water and identify a temporally isolated ${ }^{3} \mathrm{MC}$ intermediate for the first time. In contrast, we have previously demonstrated a long lived MLCT excited state (19 ps) for $\left[\mathrm{Fe}(\mathrm{bpy})(\mathrm{CN})_{4}\right]^{2-}$ in weak Lewis acidic solvents (dimethyl sulfoxide (DMSO) and acetonitrile (MeCN)), with no discernable contribution from metal centered states. ${ }^{1}$ In the present work, we shift the bpy-localized MLCT state towards higher energy by using a high Lewis acidity solvent results in a faster MLCT-toMC transition and slower MC-to-GS transition. The identification of a metastable ${ }^{3} \mathrm{MC}$ state provides further support for its role in deactivating MLCT excited states in $\mathrm{Fe}(\mathrm{II})$ complexes, and expands on the previously reported studies on Ru-centered systems which were unable to distinguish the influence of the solvent on the MC state energy levels. ${ }^{44}$

\section{Experimental methods}

\section{X-ray emission}

The time-resolved XES measurements were conducted at the X-ray Pump-Probe (XPP) ${ }^{53}$ end station at the Linac Coherent
Light Source (LCLS). An aqueous solution of $55 \mathrm{mM}\left[\mathrm{Fe}(\mathrm{bpy})(\mathrm{CN})_{4}\right]^{2-}$ was pumped through a $50 \mu \mathrm{m}$ diameter nozzle producing a cylindrical liquid jet. The sample was excited with $400 \mathrm{~nm}$ optical laser pulses of 45 fs duration, $120 \mu \mathrm{m}$ focus diameter (FWHM), and $12.5 \mu \mathrm{J}$ per pulse, delivering a pulse fluence of $3 \times 10^{12} \mathrm{~W} \mathrm{~cm}^{-2}$. The sample was probed by $8.5 \mathrm{keV}$ X-ray laser pulses of $\sim 30 \mathrm{fs}$ duration. The Fe $3 \mathrm{p}-1 \mathrm{~s}(\mathrm{~K} \beta)$ fluorescence XES signal was detected on a 140k Cornell-SLAC Pixel Array Detector (CSPAD) area detector ${ }^{54}$ located above the liquid jet using four dispersive $\mathrm{Ge}(620)$ crystal analyzers with a central Bragg angle of 79.1 degrees. ${ }^{55}$ The Fe 2p-1s (K $\left.\alpha\right)$ fluorescence XES signal was detected on a second 140k CSPAD detector placed behind the sample, using a spherically bent $\mathrm{Ge}(440)$ crystal analyzer, set to a Bragg angle corresponding to the maximum signal of the iron $\mathrm{K} \alpha$ fluorescence of $6404 \mathrm{eV}$ (75.4 degrees).

The full 2D images of the XES detectors were read out for each pump-probe event, normalized, and corrected as described in the ESI. $\dagger$ Difference images were constructed by subtracting a reference signal recorded from optical laser-off probe events for every seventh X-ray laser pulse event throughout the data collection. The difference images of each pump-probe event were then sorted into 250 individual time bins (400 shots per bin) according to their individually recorded time delay (see the ESI, $\dagger$ for a description of the timing tool diagnostic). The $\mathrm{K} \alpha$ difference images for each time bin were averaged and integrated, resulting in the kinetic trace presented in Fig. 5 , while the $\mathrm{K} \beta$ difference images of each time bin were averaged and integrated along the nondispersive detector axis, resulting in the difference spectra presented in Fig. 6A.

The static K $\alpha$ XES spectra of reference compounds were measured at the Stanford Synchrotron Radiation Lightsource (SSRL) beamline 6-2. Reference compounds were obtained from Sigma-Aldrich and used as-is. Samples were measured as powders (covered by Kapton tape) in a cryostat at $10 \mathrm{~K}$. Samples were excited with monochromatic incident X-rays at $7300 \mathrm{eV}$ (double-crystal Si(311) monochromator, 0.2 eV FWHM bandwidth) and $\mathrm{Fe} \mathrm{K} \alpha$ fluorescence was detected with a Rowland geometry spectrometer utilizing (440) Bragg reflection from bent Ge crystals with a $1 \mathrm{~m}$ radius of curvature (energy resolution $0.6 \mathrm{eV}$ FWHM) ${ }^{56}$ X-rays were detected with a Si drift detector and a correction for detector nonlinearity was applied. The monochromator energy was calibrated with an Fe foil and the spectrometer calibration and transmission correction was done using elastic scattering. Each sample was checked for X-ray induced damage by measuring the Fe X-ray absorption near-edge structure (XANES) spectra at various incident fluxes using filters installed in the beamline. Each sample was measured multiple times at different positions to reduce exposure.

\section{Optical transient absorption}

Femtosecond time-resolved UV-visible transient absorption (TA) measurements were conducted on $\left[\mathrm{Fe}(\mathrm{bpy})(\mathrm{CN})_{4}\right]^{2-}$ samples in $\mathrm{H}_{2} \mathrm{O}(7 \mathrm{mM})$ and DMSO $(2 \mathrm{mM})$, prepared directly before the experiments. Liquid sheet jets with $100 \mu \mathrm{m}$ and $200 \mu \mathrm{m}$ thicknesses were used to deliver the sample into the pumpprobe overlap region, respectively. Continuous flow of the jet 
ensured that any accumulation of photo-damaged sample was avoided.

Experiments were carried out using an amplified Ti:sapphire laser system (Coherent Mantis with Coherent Legend Elite Duo) with a $5 \mathrm{kHz}$ repetition rate, $800 \mathrm{~nm}$ central wavelength, $2 \mathrm{~mJ}$ pulse energy and $40 \mathrm{fs}$ FWHM pulse duration. A portion of the laser pumped an optical parametric amplifier (Spectra-Physics OPA-800C) to generate near IR signal and idler via difference frequency mixing. The output of the OPA was used to generate $500 \mathrm{~nm}$ pump pulses via sum-frequency generation of the signal with $800 \mathrm{~nm}$ light, and to generate $700 \mathrm{~nm}$ pump pulses via frequency doubling of the signal in a BBO crystal. The pump pulse was directed to the sample through a delay stage, a $2.5 \mathrm{kHz}$ chopper, and a lens, resulting in pump pulses with $100 \mu \mathrm{m}$ focus diameter (FWHM), $50 \mathrm{fs}$ duration, and $1.6 \mu \mathrm{J}$ pulse energy providing a pump fluence of $4 \times 10^{11} \mathrm{~W} \mathrm{~cm}^{-2}$. The pump was overlapped with a white light probe pulse (via supercontinuum generation in $4 \mathrm{~mm}$ of $\mathrm{CaF}_{2}$ ) at the sample position. The probe was transmitted through the sample and imaged on a spectrometer (Horiba Jobin Yvon iHR320, grating 150 grooves per $\mathrm{mm}$ ). The probe spectrum was recorded at $5 \mathrm{kHz}$ with a NMOS linear image sensor (Hamamatsu, S8380-512Q) simultaneously over the whole $300-700 \mathrm{~nm}$ spectral range. The differential absorbance $(\Delta A)$ was calculated as $\Delta A=\log \left(I_{\text {off }} / I_{\text {on }}\right)$, where $I_{\text {on }}$ and $I_{\text {off }}$ are the pumped and unpumped intensity, respectively. Overall time-resolution of the experiment was approximately $100 \mathrm{fs}$.

\section{Results and discussion}

\section{Steady-state and transient optical absorption spectroscopy}

The steady-state optical absorption spectrum of $\left[\mathrm{Fe}(\mathrm{bpy})(\mathrm{CN})_{4}\right]^{2-}$ in a series of solvents is presented in Fig. 1. In weak Lewis acid solvents (acetonitrile (MeCN, red), tetrahydrofuran (THF, orange), and dimethyl sulfoxide (DMSO, green)) the visible spectrum is characterized by two absorption features at roughly $450 \mathrm{~nm}$ and $700 \mathrm{~nm}$ assigned to MLCT excitations from orbitals of mixed Fe $\mathrm{d}$ and cyano $\pi$ and $\pi^{*}$ character to $\pi^{*}$ orbitals of the bipyridine ligand. ${ }^{57}$ For high Lewis acidity solvents, such as the hydrogenbonding methanol (MeOH, blue) and $\mathrm{H}_{2} \mathrm{O}$ (purple), these absorption features blue-shift. This solvatochromic effect has been extensively mapped for cyano-polypyridyl $\mathrm{Fe}$ and $\mathrm{Ru}$ complexes and shown to be linear, both with respect to the solvent acceptor number (a measure of the Lewis acidity) and the number of cyano ligands present. ${ }^{39,40,42,57}$ This makes $\left[\mathrm{Fe}(\mathrm{bpy})(\mathrm{CN})_{4}\right]^{2-}$ in solution an archetypical system to characterize the influence of the solvent Lewis acidity on the properties of these complexes.

The blue-shift of the MLCT absorption band clearly shows that increasing solvent Lewis acidity strongly destabilizes the MLCT state with respect to the ground state. To investigate how the solvent influences the energy levels of the MC states, and to determine how this modifies the MLCT deactivation pathways, we turn to the solvent dependence of the excited state relaxation dynamics.

Fig. 2 shows the solvent-dependent differential absorption spectra of $\left[\mathrm{Fe}(\mathrm{bpy})(\mathrm{CN})_{4}\right]^{2-}$ obtained after excitation of the lowest energy MLCT transition (700 nm in DMSO, $500 \mathrm{~nm}$ in water). In DMSO (Fig. 2A), the differential spectra at all time delays are characterized by the ground state bleach (GSB), overlaid with a broad excited state absorption (ESA) feature everywhere below $600 \mathrm{~nm}$, peaking at $370 \mathrm{~nm}$ and $525 \mathrm{~nm}$. In bipyridine-containing complexes, the strong $370 \mathrm{~nm}$ ESA feature is typically associated with an excited electronic state having an electron localized on the pyridyl ligand. ${ }^{1,2,23,58,59}$ In previous work, we have complemented such TA measurements by $\mathrm{K} \beta \mathrm{XES}$, determining that the excited state dynamics are well-described by a 19 ps single-exponential decay of a bipyridine-localized MLCT state. ${ }^{1}$ In water (Fig. 2B), the difference spectra at very early time delays $(\sim 100 \mathrm{fs})$ can also be described by the GSB overlaid by a similar ESA feature everywhere below
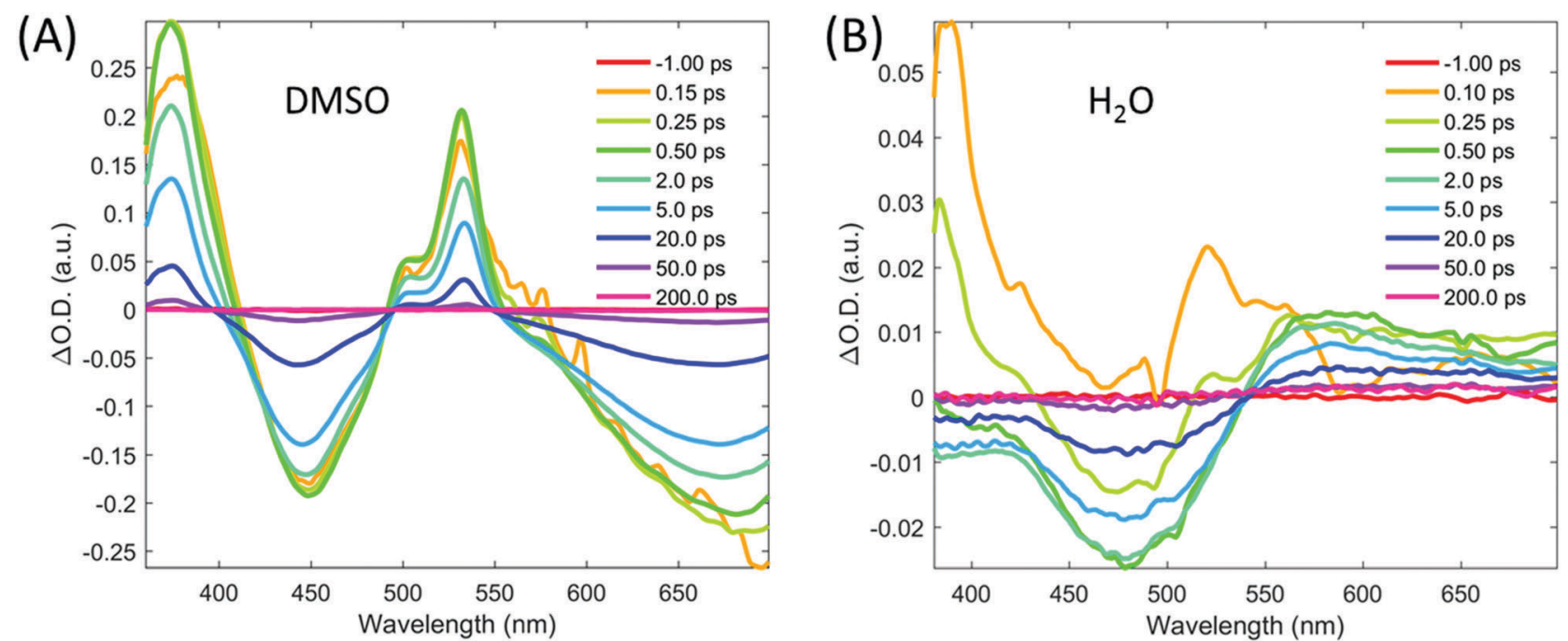

Fig. 2 Transient absorption spectra of $\left[\mathrm{Fe}(\mathrm{bpy})(\mathrm{CN})_{4}\right]^{2-}$ at selected time delays in (A) DMSO with $700 \mathrm{~nm}$ excitation and (B) water with $500 \mathrm{~nm}$ excitation. 
$600 \mathrm{~nm}$, peaking at $370 \mathrm{~nm}$ and $525 \mathrm{~nm}$. However, within half a picosecond, the initial ESA features decay completely. Differential spectra at time delays longer than 0.5 ps are characterized by the GSB and a broad ESA feature above $600 \mathrm{~nm}$. These features decay together over the following $50 \mathrm{ps}$ as the ground state spectrum recovers. For $\left[\mathrm{Fe}(\mathrm{bpy})(\mathrm{CN})_{4}\right]^{2-}$ in water, the ultrafast appearance and decay of the $370 \mathrm{~nm}$ ESA feature without the decay of the ground state bleach indicates that the excitation of the MLCT band initially populates a very short-lived bipyridine-localized MLCT state, which decays to a secondary electronic excited state before returning to the ground state on the tens of picosecond time scale. As described in the ESI, $\dagger$ high excitation fluencies lead to a longer lived absorption feature centered at $680 \mathrm{~nm}$ characteristic of the solvated electron ${ }^{60}$ arising from multiphoton ionization.

From the optical data presented in Fig. 2B, the excited state cascade in aqueous solution can be formulated as an instantaneous generation of an MLCT state and a small fraction $(<0.01)$ of photoionization products. The MLCT state undergoes ultrafast conversion to a second excited state which decays on the picosecond timescale, while the photoionization product remains. When this three-state model is implemented in a global analysis framework and applied to the data, the species associated spectra (SAS) shown in Fig. 3 are identified. SAS1, which we associate with the MLCT state, decays with a $0.17 \pm 0.03$ ps lifetime. SAS2, which we associate with the second excited electronic state, decays with a lifetime of $12.7 \pm 0.4$ ps. SAS3, which we associate with the solvated electrons remains within the $1 \mathrm{~ns}$ time window of these measurements.

To facilitate the direct comparison of the excited state dynamics measured with TA at low fluence to those measured by XES at higher fluence, we have applied the same analysis to additional TA measurements recorded at higher fluence, which exhibit a significant contribution from solvated electrons. The transient spectra and resulting SAS are shown in the ESI. $\dagger$

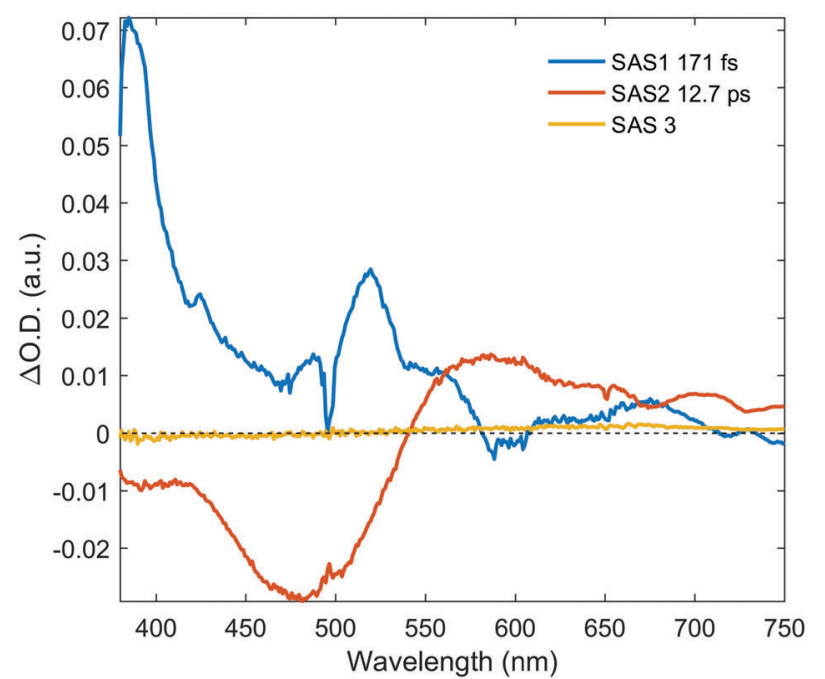

Fig. 3 Species associated spectra (SAS) from a global analysis of the transient absorption data for $\left[\mathrm{Fe}(\mathrm{bpy})(\mathrm{CN})_{4}\right]^{2-}$ dissolved in water and shown in Fig. 2B.
The spectral features of the photoexcited states (SAS1 and SAS2) are virtually identical between the high and the low fluence measurements, and the variation in the extracted relaxation dynamics lies within the uncertainties of the measurements. This demonstrates that the decay pathway of the single-photon MLCT non-radiative relaxation is independent of the excitation conditions over a large range of pump fluence.

Assigning the nature of the second electronic excited state associated with SAS2 in the global analysis is difficult based on optical TA data alone. The broad and relatively weak $>600 \mathrm{~nm}$ ESA is the only significant spectral feature outside the ground state bleach. This ESA lacks significant or defining features that might otherwise allow assignment of the associated species. The short lifetime and complete ground state recovery rules out ionization and degradation products as candidates for SAS2. The lack of low-lying unoccupied ligand-centered electronic states (other than the bipyridine $\pi^{*}$ states) suggests that SAS2 describes a secondary excited state with MC character. To unambiguously determine the nature of this intermediate, we turn to the transient $\mathrm{K} \alpha$ and $\mathrm{K} \beta$ XES measured at the LCLS $\mathrm{X}$-ray free-electron laser.

\section{Transient X-ray emission spectroscopy}

$\mathrm{K} \alpha$ and $\mathrm{K} \beta$ XES monitors the $2 \mathrm{p}$-to-1s and 3p-to-1s fluorescence, respectively, following 1 s core-hole X-ray ionization. The strong influence of the exchange interaction between the $n \mathrm{p}$ and the $3 \mathrm{~d}$ valence electrons make the XES spectra sensitive to the total spin multiplicity on the iron center. ${ }^{56,61-64}$ The spin-sensitivity of the two techniques is illustrated by the reference spectra shown in Fig. 4A and B. The reference spectra are measured for a series of Fe-centered compounds with ground state electronic structure similar to those of the potential excited states of $\left[\mathrm{Fe}(\mathrm{bpy})(\mathrm{CN})_{4}\right]^{2-}$. By selecting reference compounds with ligand bond covalency similar to $\left[\mathrm{Fe}(\mathrm{bpy})(\mathrm{CN})_{4}\right]^{2-}$, good agreement between the reference spectra and the measured data is expected. By subtracting the reference signal of the ground state compound, from the references for the potential excited states, the reference difference spectra, illustrated in Fig. 4C and D can be constructed.

The $\mathrm{K} \beta$ spectra exhibit the clearest spectral shape variation between different electronic states, such that direct comparison between transient $K \beta$ data and reference difference spectra allows for robust identification of the excited electronic states. Meanwhile, the $\mathrm{K} \alpha$ emission is roughly an order of magnitude more intense than the $\mathrm{K} \beta$ emission, which makes the $\mathrm{K} \alpha$ data well suited to derive the time scales of the underlying relaxation dynamics. Based on these considerations, we have measured $\mathrm{K} \alpha$ single-energy kinetics to determine the characteristic time scales of the experiment (time-zero, instrument-response function (IRF), and lifetimes), and measured full $\mathrm{K} \beta$ transient spectra for robust excited state identification. Since the $\mathrm{K} \alpha$ and $\mathrm{K} \beta$ data were recorded simultaneously, the time zero, temporal resolution, and quantum yields of the two datasets are identical. Therefore, the characteristic time constants of the experimental parameters, as well as the excited state lifetimes, determined from the high signal-to-noise $\mathrm{K} \alpha$ kinetic data can be used in a global analysis framework for the $K \beta$ spectral data. 

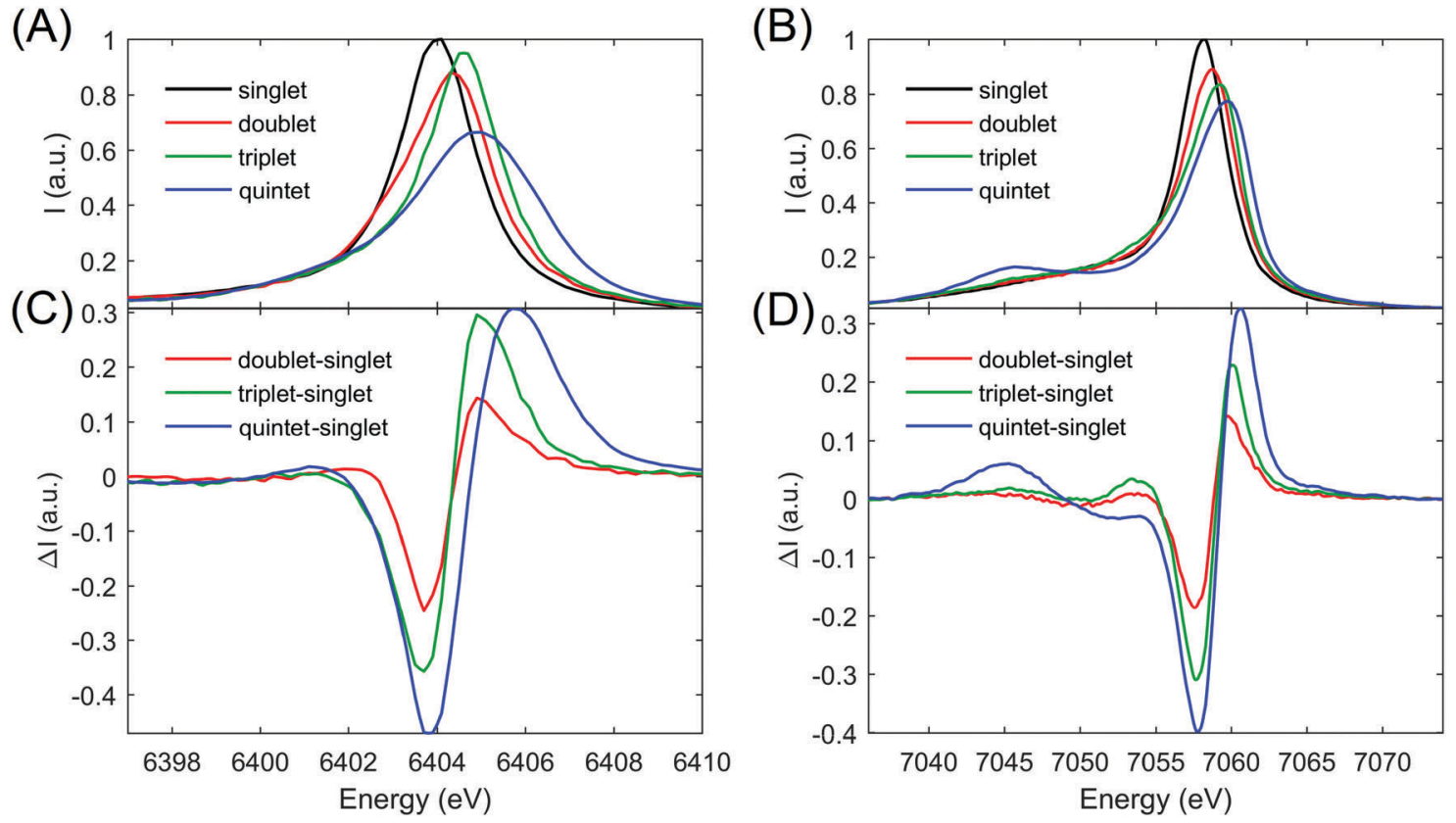

Fig. $4 K \alpha_{1}(A)$ and $K \beta(B)$ reference spectra measured for a series of Fe model complexes of similar spin state configuration as the potential excited states in this study. $\mathrm{K} \alpha$ references are $\left[\mathrm{Fe}(\mathrm{bpy})(\mathrm{CN})_{4}\right]^{2-}\left(\right.$ singlet), $\left[\mathrm{Fe}(\mathrm{CN})_{6}\right]^{3-}$ (doublet), Fe(phthalocyanine) (triplet), and $\left[\mathrm{Fe}(1,10-\text { phenanthroline })_{2}(\mathrm{NCS})_{2}\right]$ (quintet). $\mathrm{K} \beta$ references are taken from Zhang et al. ${ }^{1}$ Reference difference spectra ( $C$ and $\left.D\right)$ constructed from the K $\alpha$ and $K \beta$ references generated by subtracting the ground state reference from each of the excited state references.

The $\mathrm{K} \alpha$ kinetics shown in Fig. 5 were recorded at $6404 \mathrm{eV}$. As reported elsewhere, ${ }^{62}$ and seen from the reference difference spectra (Fig. 4C), the K $\alpha$ difference intensity at $6404 \mathrm{eV}$ allow us to monitor the total spin increase on the Fe center. We observe that the growth of the transient signal is delayed with respect to a broadened Heaviside step function. This delay arises from an

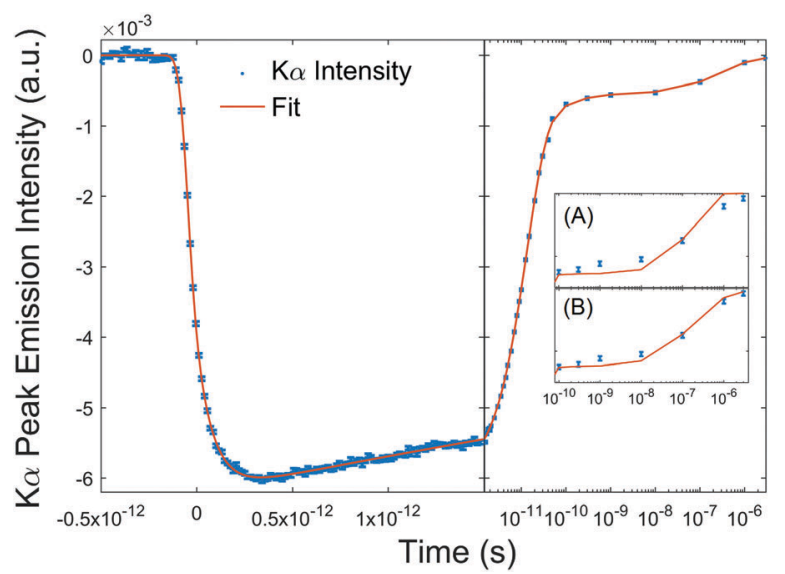

Fig. 5 Kinetics of the $K \alpha_{1}$ peak emission intensity measured at $6404 \mathrm{eV}$ following MLCT excitation of $\left[\mathrm{Fe}(\mathrm{bpy})(\mathrm{CN})_{4}\right]^{2-}$ in water. Blue data points are binned in $10 \mathrm{fs}$ steps and supplied with error bars indicating the standard error. The red curve shows the fit of the data as described in the main text. Insets (A) and (B) show fits to the late data points using first and second order reaction kinetics, respectively. The second order reaction kinetics $(B)$, capture the evolution of the data better, illustrating that the kinetics are due to charge-recombination of photoionized $\left[\mathrm{Fe}(\mathrm{bpy})(\mathrm{CN})_{4}\right]^{-}$with solvated electrons, as described in detail in the ESI. $\dagger$ excited state relaxation that results in a secondary increase in the Fe spin-moment, delayed with respect to excitation of the MLCT state. Overall, the growth is best described by the sum of a Heaviside step function and exponential grow-in broadened by a Gaussian representing the instrument-response function. The step function accounts for the instantaneously populated ${ }^{1}$ MLCT and photoionization products. ${ }^{65,66}$ These species each have a single unpaired electron on the Fe center, making them formal doublets with respect to the Fe center, and therefore they cannot be distinguished with the XES data. The exponential growth accounts for the transition from the MLCT to the secondary excited state of higher spin-moment. The fact that the Fe spin-moment increases for the secondary excited state confirms the assumption, based on the optical TA data, that the decay of the MLCT state leads to the population of an MC state with higher spin. The decay of the transient $\mathrm{K} \alpha$ signal is dominated by the single-exponential lifetime of the secondary MC excited state, but also includes a weaker long time-scale component for the recovery of the photoionization products.

In fitting the data to the kinetic model described above, a time-zero and IRF (rms) of $-54 \pm 4$ fs and $34 \pm 2$ fs are recovered, with the uncertainty reflecting the standard deviation. The exponential component of the growth accounts for a $40 \pm 4 \%$ increase of the total signal and has a time constant of $87 \pm 5 \mathrm{fs}$, which we assign to the MLCT-to-MC transition. The decay is dominated by a component with a $13.1 \pm 0.4 \mathrm{ps}$ lifetime, readily assigned to the decay of the MC state and recovery of the ground state accounting for $84 \%$ of the decay. The remaining decay of the transient signal occurs on the hundreds-ofnanoseconds to microsecond time scale. The MLCT-to-MC 
transition observed by the XES is faster than the lifetime associated with SAS1 in the TA analysis. This discrepancy likely reflects the challenge of differentiating intramolecular vibrational relaxation and population decay in TA, combined with the challenges of properly subtracting out cross-phase modulation artefacts.

The long time scale dynamics of the ground state recovery matches the expected second-order kinetics (as described in the $\mathrm{ESI}+$ ) for the recovery from the photoionized $\left[\mathrm{Fe}(\mathrm{bpy})(\mathrm{CN})_{4}\right]^{-60}$ Comparative fits to the long time delay data using first- and second order rate expressions for the decay are illustrated in Fig. 5 as insets (A) and (B), respectively. A detailed discussion of the mechanism and time scale of the recovery can be found in the ESI. $\dagger$ The increased ratio of photoionization product (16\%) compared to the TA data is due to the increased laser power used for the XES experiments.

In summary, the $\mathrm{K} \alpha$ decay kinetics can be described by the same model used to interpret the TA data. Since the TA experiment shows that the dynamics of the single-photon excited state pathway is independent on the amount of twophoton excitation, the XES data can be used to identify the spin state of the 13 ps MC intermediate associated with SAS2 in the TA analysis. For this excited state identification, we rely on the $\mathrm{K} \beta$ data recorded simultaneously with the $\mathrm{K} \alpha$ data.

Transient $\mathrm{K} \beta$ differential spectra measured at multiple time delays are presented in Fig. 6A. Qualitative comparison of the data recorded at $0.5-5$ ps (delays dominated by the MC intermediate) with the reference spectra in Fig. 4D reveals that the $\mathrm{K} \beta^{\prime}$ shoulder at $7045 \mathrm{eV}$ is very weak. This allows the high spin ${ }^{5} \mathrm{MC}$ state to be eliminated as the dominant product of the MLCT decay and makes a ${ }^{3} \mathrm{MC}$ the most likely candidate for the MC excited state. To explicitly identify the nature of the MC state and derive excitation and speciation fractions, we have conducted a global analysis of the transient $\mathrm{K} \beta$ data using a three-state model that includes the MLCT state, the MC state, and the $\left[\mathrm{Fe}(\mathrm{bpy})(\mathrm{CN})_{4}\right]^{-}$photoproduct. Using the excited state cascade and time scales from the $\mathrm{K} \alpha$ analysis, the species associated spectra (SAS) for the excited states were extracted.

The extracted SAS are shown in Fig. 6B. SAS1 describes the signal that is lost with the 85 fs time constant after the excitation. SAS2 describes the signal that grows in as SAS1 decays, and has a lifetime of 13.1 ps. SAS1 was definitively identified as an MLCT state from the optical TA, and could therefore be used to establish a scaling factor between the SAS and reference difference spectra by minimizing the chi-squared between SAS1 and the MLCT (doublet-singlet) reference difference signal. Upon establishing this scaling factor, a quantitative comparison of SAS2 (representing the MC excited state) can be made against the triplet and quintet reference difference spectra (all plotted in Fig. 6B). It is immediately apparent that the signal shape, and in particular signal amplitude, of SAS2 matches the reference spectrum obtained for the ${ }^{3} \mathrm{MC}$ state. Taken together with the lack of additional fast time constants in the excited state cascade, the agreement between SAS2 and the ${ }^{3} \mathrm{MC}$ reference difference spectrum definitively assigns the ultrafast interconversion observed in the optical TA and $\mathrm{K} \alpha$ data to an MLCT-to- ${ }^{3} \mathrm{MC}$ interconversion. Furthermore, the spectral features of SAS2 in the optical TA analysis can now be assigned as characteristic of the ${ }^{3} \mathrm{MC}$ state. The ultrafast XES measurements provide a robust assignment of the ${ }^{3} \mathrm{MC}$ state, which enables the interpretation of the ESA seen in the optical pump-probe measurement. The most significant feature of the spectrum is the excited state absorption feature at $570 \mathrm{~nm}$, to the red side of the ground state bleach. Similar, but somewhat weaker, ESA features have been observed for ${ }^{3} \mathrm{MC}$ states assigned of Ru-centered polypyridyl complexes. ${ }^{67}$

\section{Solvent-dependent electronic structure}

The solvent Lewis acidity strongly influences the excited state dynamics of $\left[\mathrm{Fe}(\mathrm{bpy})(\mathrm{CN})_{4}\right]^{2-}$. Increasing solvent Lewis acidity
(A)

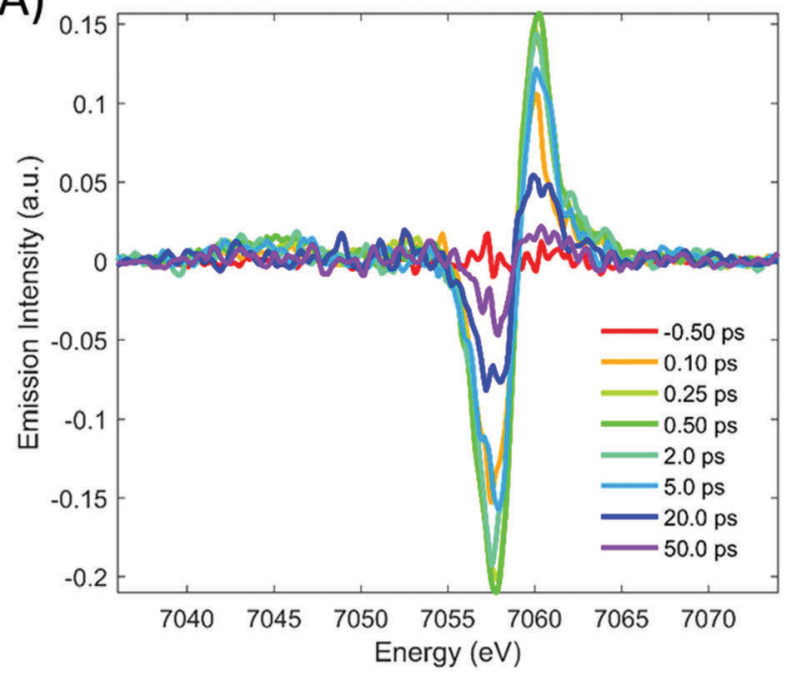

(B)

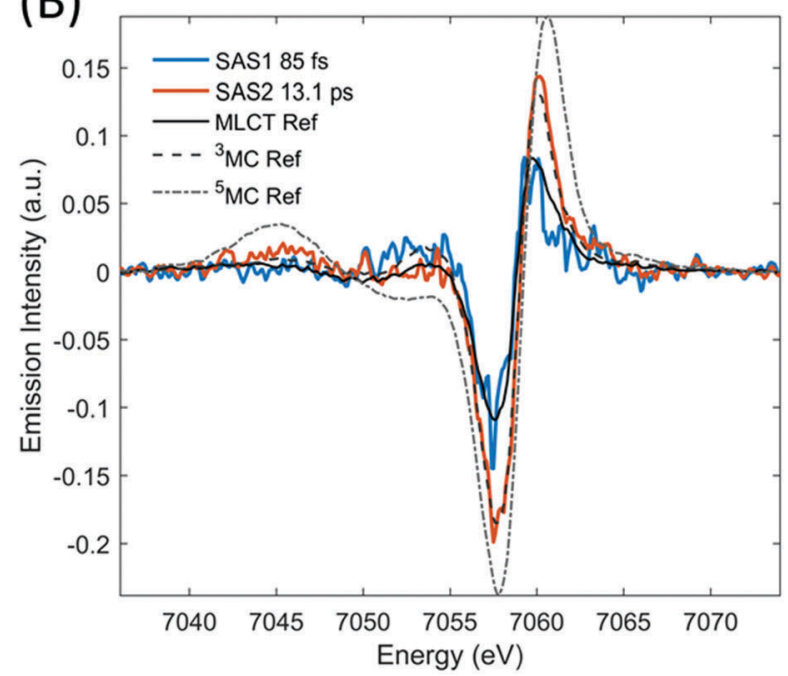

Fig. 6 Transient $K \beta$ spectra and analysis. (A) Transient $K \beta$ emission signal of $\left[\mathrm{Fe}(\mathrm{bpy})(\mathrm{CN})_{4}\right]^{2-}$ in water. (B) Species associated spectra (SAS, red and blue) from a global analysis, invoking the model used for fitting the TA and K $\alpha$ kinetics and the reference difference spectra of Fig. 4D scaled by mapping the MLCT reference to SAS1. 
leads to rapid excited state interconversion of the otherwise relatively long lived MLCT state. Furthermore, the ${ }^{3} \mathrm{MC}$ intermediate, which is expected to govern the MLCT decay, is stabilized to the point where it can be considered a wellisolated intermediate in the non-radiative relaxation. These changes in excited state dynamics reflect the solvent-dependent changes in the electronic structure of $\left[\mathrm{Fe}(\mathrm{bpy})(\mathrm{CN})_{4}\right]^{2-}$. A qualitative molecular orbital (MO) diagram of $\left[\mathrm{Fe}(\mathrm{bpy})(\mathrm{CN})_{4}\right]^{2-}$ in weak Lewis acid solvent is shown in Fig. 7A. Both $t_{2 g}$ and $e_{g}$ levels of the cyano-pyridyl complex increase upon substitution of bpy with $\mathrm{CN}^{-}$ ligands, as demonstrated by the reduction in the metal oxidation potential, the reduction in the MLCT excitation energy, and the elimination of the quintet state from the excited state relaxation pathway. ${ }^{1,2,39,44}$ These energetic changes in the redox potentials are readily explained by the $\sigma$ and $\pi^{*}$ energy levels of the $\mathrm{CN}^{-}$ ligand being of higher than the $\sigma$ and $\pi^{*}$ levels of bpy, as illustrated schematically in Fig. 7A. This MO picture is consistent with the projected potential energy surfaces (PES), calculated for $\left[\mathrm{Fe}(\mathrm{bpy})(\mathrm{CN})_{4}\right]^{2-}$ in DMSO, which are illustrated in a schematic form in Fig. 7B, based on the calculations in Zhang et al. ${ }^{1}$

Increasing solvent Lewis acidity leads to a blue-shift of the MLCT absorption bands, as shown in Fig. 1. A similar solvation response has also been observed for Ru-centered cyano-pyridyls, where the solvent-dependent blue-shift has been shown to increase linearly with both the number of $\mathrm{CN}^{-}$ligands and the Lewis acidity of the solvent. ${ }^{39}$ The blue-shift is interpreted as a result of the high Lewis acidity solvent shifting electron density of the $\mathrm{CN}^{-}$orbitals away from the metal center, decreasing the $\pi\left(\mathrm{CN}^{-}\right) \rightarrow \mathrm{d}(\mathrm{Fe}) \pi$-bonding and increasing the $\mathrm{d}(\mathrm{Fe}) \rightarrow \pi^{*}\left(\mathrm{CN}^{-}\right)$ $\pi$-backbonding. Both of these changes stabilize the Fe $t_{2 g}$ levels relative to the (poly)pyridyl centered $\pi^{*}$ levels (which are relatively unaffected by solvation).

The influence of lowering the $t_{2 g}$ levels on the PES is illustrated in Fig. 7C. The GS having $6 t_{2 g}$ electrons is stabilized more than the MLCT and the ${ }^{3} \mathrm{MC}$ states each having $5 \mathrm{t}_{2 \mathrm{~g}}$ electrons, which are in turn stabilized more than the ${ }^{5} \mathrm{MC}$ state having only $4 \mathrm{t}_{2 \mathrm{~g}}$ electrons. Since the GS is stabilized the most, all excited states undergo an apparent destabilization since the PES in Fig. 7C are normalized relative to the ground state energy. Since the ${ }^{5} \mathrm{MC}$ state has two less $\mathrm{t}_{2 \mathrm{~g}}$ electrons than the GS, and the ${ }^{3} \mathrm{MC}$ and MLCT states have one less $\mathrm{t}_{2 \mathrm{~g}}$ electron than the GS, the relative destabilization of the ${ }^{5} \mathrm{MC}$ state is twice that of the ${ }^{3} \mathrm{MC}$ and MLCT states.

While the changes in the $t_{2 g}$ energy levels can be addressed by steady state measurements, the changes in $e_{g}$ levels are most clearly addressed with transient measurements since the $e_{g}$ levels only influence the metal centered electronic excited states. To explain the significant shortening of the MLCT lifetime of $\left[\mathrm{Fe}(\mathrm{bpy})(\mathrm{CN})_{4}\right]^{2-}$ in water relative to DMSO, the ${ }^{3} \mathrm{MC}$ state has to intersect the MLCT surface closer to its energy minimum. Therefore, the ${ }^{3} \mathrm{MC}$ state must be stabilized relative to the MLCT state, which indicates that the $e_{g}$ levels also decrease in energy with increasing solvent Lewis acidity. This stabilization of the $\sigma$-bonded $e_{\mathrm{g}}$ orbitals is rationalized by the

\section{(A)}

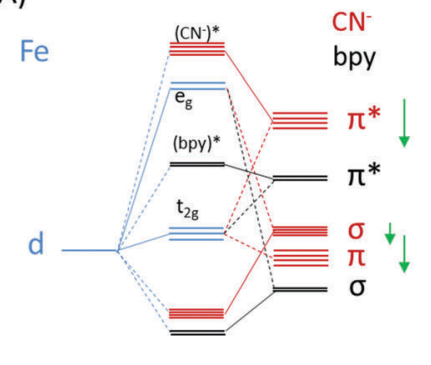

(B)

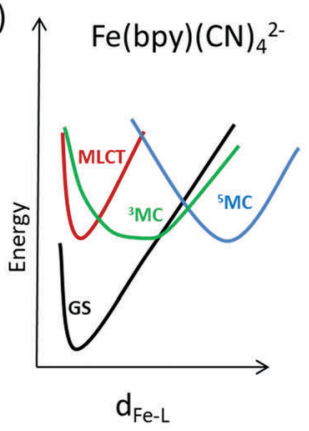

(C)
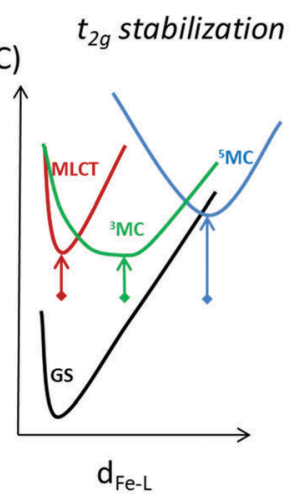

(D) $t_{2 g}>e_{g}$ stabilization

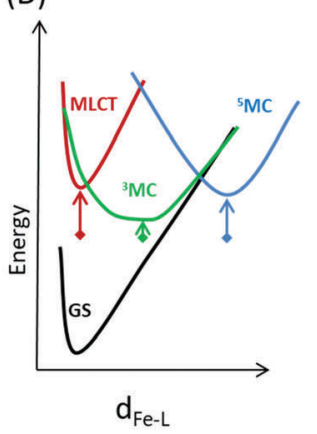

(E)

$$
t_{2 g}<e_{g} \text { stabilization }
$$

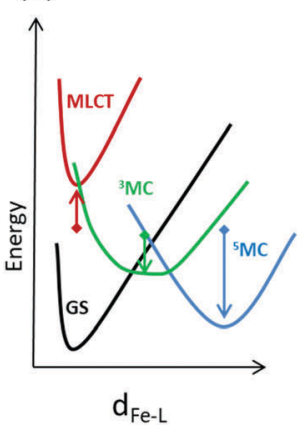

Fig. 7 (A) Schematic molecular orbital diagram for $\left[\mathrm{Fe}(\mathrm{bpy})(\mathrm{CN})_{4}\right]^{2-}$. Green arrows indicate the shift in energy levels upon solvation in high Lewis acidity solvents. (B) Schematic of the potential energy surfaces of $\left[\mathrm{Fe}(\mathrm{bpy})(\mathrm{CN})_{4}\right]^{2-}$ in low Lewis acidity solvents. ${ }^{1}(\mathrm{C}-\mathrm{E})$ Effect of variable $\mathrm{t}_{2 \mathrm{~g}}$ and $\mathrm{e}_{\mathrm{g}}$ orbital stabilization on the MLCT and MC excited state PES. 
same argument for the $\pi$-bonded $t_{2 g}$ orbital stabilization discussed above. Stabilization of the $e_{g}$ orbitals will shift down the ${ }^{3} \mathrm{MC}$ and ${ }^{5} \mathrm{MC}$ states relative to the illustration in Fig. $7 \mathrm{C}$, which only accounts for the $t_{2 g}$ orbital stabilization. Two cases accounting for both $t_{2 g}$ and $e_{g}$ stabilization are illustrated in Fig. 7D and E.

Stabilization of the $\mathrm{e}_{\mathrm{g}}$ orbitals is consistent with the solvent dependence of the MLCT lifetimes in Ru-centered cyanopyridyls. ${ }^{44}$ For these $\mathrm{Ru}$ complexes, the relationship between MLCT lifetime and solvent acceptor number (a measure of the solvent Lewis acidity) could be described by assuming that the ${ }^{3} \mathrm{MC}$ energy was unchanged relative to the GS, requiring identical stabilization of the $t_{2 g}$ and the $e_{g}$ levels. Interestingly, our experiments show that the ${ }^{3} \mathrm{MC}$ lifetime, which is too short to be clearly observed in low Lewis acidity solvents, ${ }^{1}$ is significantly extended in high Lewis acidity solvents, meaning that a reaction barrier for the inter-system crossing and internal conversion back to the GS is required. This reaction barrier can only arise by stabilizing the $\mathrm{e}_{\mathrm{g}}$ levels by a smaller amount than the $t_{2 \mathrm{~g}}$ levels, such that the MC states are destabilized relative to the GS (Fig. 7D). If the $\mathrm{e}_{\mathrm{g}}$ levels are destabilized equal to or more than the $t_{2 g}$ levels, then the MC states are stabilized with respect to the GS, as illustrated in Fig. 7E. In this case, it becomes impossible to arrange the potential energy surfaces such that there is a reaction barrier to the ground state, while maintaining a significant reaction barrier to the ${ }^{5} \mathrm{MC}$ state consistent with the stabilization of the ${ }^{3} \mathrm{MC}$ and ${ }^{5} \mathrm{MC}$ states being proportionally with their number of $e_{g}$ electrons. Since we observe no signature of the ${ }^{5} \mathrm{MC}$ state in the excited state cascade, we conclude the ordering of the potential energy surfaces of $\left[\mathrm{Fe}(\mathrm{bpy})(\mathrm{CN})_{4}\right]^{2-}$ in high Lewis acidity solvents match the illustration in Fig. 7D. In summary, the observation that the MLCT lifetime decreases with increasing solvent Lewis acidity shows that the $\mathrm{e}_{\mathrm{g}}$ levels are stabilized in strong Lewis acid solvents, but not as strongly as the $t_{2 g}$ levels.

To support our interpretation of the solvent influence on the excited state energy levels, we have performed preliminary DFT calculations with a polarizable continuum description of both water and DMSO, and by including explicit water molecules at the $\mathrm{N}$ lone-pair site of the $\mathrm{CN}^{-}$groups. The effects of implicit and explicit solvent interactions on the excited state energy levels are summarized in Table S1 in the ESI. $\dagger$ It is observed that there is virtually no difference between the energy levels calculated for water and DMSO when representing the solvents with continuum models. However, upon inclusion of the explicit waters at the $\mathrm{N}$ lone pair sites of the $\mathrm{CN}^{-}$groups, the DFT calculations shows a destabilization of ${ }^{3} \mathrm{MLCT},{ }^{5} \mathrm{MC}$, and ${ }^{3} \mathrm{MC}$ states with respect to the ground state, in parallel to our experimental observations. In accordance with our interpretation of the experimental results, the calculations also show that the ${ }^{3} \mathrm{MLCT}$ state is destabilized the most, and the ${ }^{3} \mathrm{MC}$ state is destabilized the least. The minimum energy structures of our calculations (presented in Fig. S5, ESI $\dagger$ ) shows that the water orients to form hydrogen bonds between to the cyanide $\mathrm{N}$ lone-pairs, supporting our interpretation of the experimental results that the reordering of the excited state level arises from explicit electronic interactions between solute and solvent.

\section{Closing remarks}

Combined ultrafast optical and XES measurements provide a clear picture for the MLCT excited state relaxation dynamics in $\left[\mathrm{Fe}(\mathrm{bpy})(\mathrm{CN})_{4}\right]^{2-}$. In high Lewis acidity solvents, coordination of the $\mathrm{CN}^{-}$ligands to the solvent significantly changes the lifetimes of the MLCT and the ${ }^{3} \mathrm{MC}$ excited states relative to their lifetimes in low Lewis acidity solvents. The influence of the solvent on the excited state relaxation dynamics arise from inequivalent solvent stabilization of the $\mathrm{CN}^{-}$and bpy energy levels, shifting the $\mathrm{CN}^{-}$-influenced $t_{2 g}$ and $e_{g}$ orbitals down relative to the bpy $\pi^{*}$ levels, which in turn modifies the relative energies of the MLCT, ${ }^{3} \mathrm{MC}$, and ${ }^{5} \mathrm{MC}$ excited state potential energy surfaces. Our study presents a clear identification and characterization of a metastable Fe-centered ${ }^{3} \mathrm{MC}$ state in a hexacoordinated Fe centered complex.

Extracting this level of detail about the excited state relaxation mechanism has been made possible by the combined application of time resolved UV-visible TA and XES. Combining these techniques has allowed us to address not only the MLCT state dynamics, but also to identify the role of MC excited states in the electronic excited state relaxation, obtaining a more detailed picture of the excited state potential energy surfaces involved in the relaxation process. The increased robustness of the relaxation mechanisms extracted from the combined analysis of XES and TA measurements make them more amenable targets for computational quantum dynamics studies. Moreover, the relative simplicity of the Fe cyano-pyridyl compounds, and the fact that their potential energy landscape can be extensively modified by the choice of solvent, enabled a systematic exploration of the relationship between the potential energy landscape and the excited state lifetime and relaxation pathways.

These experiments also act as a stepping stone towards the investigation of Fe-centered photoactive molecular systems. With regards to future XES measurements, the good agreement with our reference spectra and the difference signal extracted from global analysis, provide further support for using the XES technique to identify the excited state species in $3 \mathrm{~d}$ transition metal centered systems. With regards to future TA measurements, the clearly identified and isolated ${ }^{3} \mathrm{MC}$ state presented here exhibit significant excited state absorption features throughout the visible spectrum. This should significantly help the assignment of excited state absorptions in future TA measurements on chemically related Fe coordination complexes.

\section{Conflicts of interest}

There are no conflicts to declare.

\section{Acknowledgements}

Work by K. K., A. A. C, and K. J. G was supported by the U.S. Department of Energy, Office of Science, Basic Energy Sciences, Chemical Sciences, Geosciences, and Biosciences Division. KL acknowledges a Melvin and Joan Lane Stanford Graduate Fellowship. KSK, KH, MMN, MGL, PV, EB, FBH, MC gratefully 
acknowledge DANSCATT support for the beamtime efforts. TCBH, EB, MGL, and MMN gratefully acknowledge support by the Danish Council for independent Research under grant no. DFF-4002-00272B. KSK gratefully acknowledge the support of the Carlsberg Foundation and the Danish Council for Independent Research. This project was supported by the 'Lendület' (Momentum) Program of the Hungarian Academy of Sciences (LP2013-59), the Government of Hungary and the European Regional Development Fund under grant No. VEKOP2.3.2-16-2017-00015, the European Research Council via contract ERC-StG-259709 (X-cited!), and the Hungarian Scientific Research Fund (OTKA) under contract K 109257. ZN acknowledges support from the Bolyai Fellowship of the Hungarian Academy of Sciences. The ELI-ALPS project (GINOP-2.3.615-2015-00001) is supported by the European Union and co-financed by the European Regional Development Fund. JU acknowledges support from the Knut and Alice Wallenberg Foundation (KAW). SK acknowledges the support from Knut \& Alice Wallenberg foundation (KAW 2014.0370). MER acknowledges a Swiss National Science Foundation fellowship, project number 158890. Use of the Stanford Synchrotron Radiation Lightsource, SLAC National Accelerator Laboratory, is supported by the U.S. Department of Energy, Office of Science, Office of Basic Energy Sciences under Contract No. DE-AC0276SF00515. Use of the Linac Coherent Light Source (LCLS), SLAC National Accelerator Laboratory, is supported by the U.S. Department of Energy, Office of Science, Office of Basic Energy Sciences under Contract No. DE-AC02-76SF00515.

\section{References}

1 W. Zhang, et al., Manipulating charge transfer excited state relaxation and spin crossover in iron coordination complexes with ligand substitution, Chem. Sci., 2016, 8, 515-523.

2 Y. Liu, et al., A Heteroleptic Ferrous Complex with Mesoionic Bis(1,2,3-triazol-5-ylidene) Ligands: Taming the MLCT Excited State of Iron(II), Chem. - Eur. J., 2015, 21, 3628-3639.

3 J. He, B. Wang, S. Chang and T. Chen, Ruthenium-Based Photosensitizers for Dye-Sensitized Solar Cells, in Organometallics and Related Molecules for Energy Conversion, ed. W.-Y. Wong, Springer, Berlin Heidelberg, 2015, pp. 91-114, DOI: 10.1007/978-3-662-46054-2_4.

4 E. Baranoff, First-Row Transition Metal Complexes for the Conversion of Light into Electricity and Electricity into Light, in Organometallics and Related Molecules for Energy Conversion, ed. W.-Y. Wong, Springer, Berlin Heidelberg, 2015, pp. 61-90, DOI: 10.1007/978-3-662-46054-2_3.

5 J. V. Caspar and T. J. Meyer, Photochemistry of tris(2,2'bipyridine)ruthenium $(2+)$ ion ( $\mathrm{Ru}($ bpy $) 32+)$. Solvent effects, J. Am. Chem. Soc., 1983, 105, 5583-5590.

6 A. Juris, et al., Ru(II) polypyridine complexes: photophysics, photochemistry, electrochemistry, and chemiluminescence, Coord. Chem. Rev., 1988, 84, 85-277.

7 N. H. Damrauer, et al., Femtosecond Dynamics of ExcitedState Evolution in $[\mathrm{Ru}(\mathrm{bpy}) 3]^{2+}$, Science, 1997, 275, 54-57.
8 M. Abrahamsson, et al., A $3.0 \mu$ s Room Temperature Excited State Lifetime of a Bistridentate RuII-Polypyridine Complex for Rod-like Molecular Arrays, J. Am. Chem. Soc., 2006, 128, 12616-12617.

9 D. G. Brown, N. Sanguantrakun, B. Schulze, U. S. Schubert and C. P. Berlinguette, Bis(tridentate) RutheniumTerpyridine Complexes Featuring Microsecond ExcitedState Lifetimes, J. Am. Chem. Soc., 2012, 134, 12354-12357.

10 S. Nozawa, et al., Direct Probing of Spin State Dynamics Coupled with Electronic and Structural Modifications by Picosecond Time-Resolved XAFS, J. Am. Chem. Soc., 2010, 132, 61-63.

11 J. E. Monat and J. K. McCusker, Femtosecond Excited-State Dynamics of an Iron(II) Polypyridyl Solar Cell Sensitizer Model, J. Am. Chem. Soc., 2000, 122, 4092-4097.

12 H. T. Lemke, et al., Femtosecond X-ray Absorption Spectroscopy at a Hard X-ray Free Electron Laser: Application to Spin Crossover Dynamics, J. Phys. Chem. A, 2013, 117, 735-740.

13 N. Huse, et al., Femtosecond Soft X-ray Spectroscopy of Solvated Transition-Metal Complexes: Deciphering the Interplay of Electronic and Structural Dynamics, J. Phys. Chem. Lett., 2011, 2, 880-884.

14 A. Cannizzo, et al., Light-induced spin crossover in Fe(II)-based complexes: The full photocycle unraveled by ultrafast optical and X-ray spectroscopies, Coord. Chem. Rev., 2010, 254, 2677-2686.

15 K. Haldrup, et al., Observing Solvation Dynamics with Simultaneous Femtosecond X-ray Emission Spectroscopy and X-ray Scattering, J. Phys. Chem. B, 2016, 120, 1158-1168.

16 N. Huse, et al., Photo-Induced Spin-State Conversion in Solvated Transition Metal Complexes Probed via TimeResolved Soft X-ray Spectroscopy, J. Am. Chem. Soc., 2010, 132, 6809-6816.

17 M. Khalil, et al., Picosecond X-ray Absorption Spectroscopy of a Photoinduced Iron(II) Spin Crossover Reaction in Solution, J. Phys. Chem. A, 2006, 110, 38-44.

18 G. Auböck and M. Chergui, Sub-50 fs photoinduced spin crossover in [Fe(bpy)3]2 $2^{+}$, Nat. Chem., 2015, 7, 629-633.

19 J. K. McCusker, et al., Subpicosecond 1MLCT.fwdarw. 5T2 intersystem crossing of low-spin polypyridyl ferrous complexes, J. Am. Chem. Soc., 1993, 115, 298-307.

20 A. Marino, et al., The Role of Ligand-Field States in the Ultrafast Photophysical Cycle of the Prototypical Iron(II) Spin-Crossover Compound [Fe(ptz)6](BF4)2, Angew. Chem., Int. Ed., 2014, 53, 3863-3867.

21 W. Zhang, et al., Tracking excited-state charge and spin dynamics in iron coordination complexes, Nature, 2014, 509, 345-348.

22 C. Sousa, et al., Ultrafast Deactivation Mechanism of the Excited Singlet in the Light-Induced Spin Crossover of [Fe(2,2'-bipyridine)3 $]^{2+}$, Chem. - Eur. J., 2013, 19, 17541-17551.

23 W. Gawelda, et al., Ultrafast Nonadiabatic Dynamics of [FeII(bpy)3]2+ in Solution, J. Am. Chem. Soc., 2007, 129, 8199-8206.

24 C. Consani, et al., Vibrational Coherences and Relaxation in the High-Spin State of Aqueous [FeII(bpy)3]2+, Angew. Chem., Int. Ed., 2009, 48, 7184-7187. 
25 Y. Liu, et al., Towards longer-lived metal-to-ligand charge transfer states of iron(II) complexes: an N-heterocyclic carbene approach, Chem. Commun., 2013, 49, 6412-6414.

26 L. A. Fredin, et al., Exceptional Excited-State Lifetime of an Iron(II)-N-Heterocyclic Carbene Complex Explained, J. Phys. Chem. Lett., 2014, 5, 2066-2071.

27 T. C. B. Harlang, et al., Iron sensitizer converts light to electrons with 92\% yield, Nat. Chem., 2015, 7, 883-889.

28 T. Duchanois, et al., An Iron-Based Photosensitizer with Extended Excited-State Lifetime: Photophysical and Photovoltaic Properties, Eur. J. Inorg. Chem., 2015, 2469-2477.

29 M. Pápai, G. Vankó, T. Rozgonyi and T. J. Penfold, High-Efficiency Iron Photosensitizer Explained with Quantum Wavepacket Dynamics, J. Phys. Chem. Lett., 2016, 7, 2009-2014.

30 Y. Liu, P. Persson, V. Sundström and K. Wärnmark, Fe N-Heterocyclic Carbene Complexes as Promising Photosensitizers, Acc. Chem. Res., 2016, 49, 1477-1485.

31 L. Liu, et al., A new record excited state 3MLCT lifetime for metal organic iron(II) complexes, Phys. Chem. Chem. Phys., 2016, 18, 12550-12556.

32 M. Reinhard, et al., Photoaquation Mechanism of Hexacyanoferrate(II) Ions: Ultrafast 2D UV and Transient Visible and IR Spectroscopies, J. Am. Chem. Soc., 2017, 139, 7335-7347.

33 C. D. Graaf and C. Sousa, On the role of the metal-to-ligand charge transfer states in the light-induced spin crossover in FeII (bpy)3, Int. J. Quantum Chem., 2011, 111, 3385-3393.

34 A. Moguilevski, et al., Ultrafast Spin Crossover in $\left[\mathrm{FeII}(\mathrm{bpy})_{3}\right]^{2+}$ : Revealing Two Competing Mechanisms by Extreme Ultraviolet Photoemission Spectroscopy, ChemPhysChem, 2017, 18, 465-469.

35 I. M. Dixon, F. Alary, M. Boggio-Pasqua and J.-L. Heully, Reversing the relative $3 \mathrm{MLCT}-3 \mathrm{MC}$ order in Fe(II) complexes using cyclometallating ligands: a computational study aiming at luminescent $\mathrm{Fe}(\mathrm{II})$ complexes, Dalton Trans., 2015, 44, 13498-13503.

36 L. L. Jamula, A. M. Brown, D. Guo and J. K. McCusker, Synthesis and Characterization of a High-Symmetry Ferrous Polypyridyl Complex: Approaching the 5T2/3T1 Crossing Point for FeII, Inorg. Chem., 2014, 53, 15-17.

37 I. M. Dixon, F. Alary, M. Boggio-Pasqua and J.-L. Heully, The (N4C2)2- Donor Set as Promising Motif for Bis(tridentate) Iron(II) Photoactive Compounds, Inorg. Chem., 2013, 52, 13369-13374.

38 M. Pápai, T. J. Penfold and K. B. Møller, Effect of tert-Butyl Functionalization on the Photoexcited Decay of a $\mathrm{Fe}(\mathrm{II})-\mathrm{N}$ Heterocyclic Carbene Complex, J. Phys. Chem. C, 2016, 120, 17234-17241.

39 C. J. Timpson, C. A. Bignozzi, B. P. Sullivan, E. M. Kober and T. J. Meyer, Influence of Solvent on the Spectroscopic Properties of Cyano Complexes of Ruthenium(II), J. Phys. Chem., 1996, 100, 2915-2925.

40 I. Georgieva, A. J. A. Aquino, N. Trendafilova, P. S. Santos and H. Lischka, Solvatochromic and Ionochromic Effects of Iron(II)bis(1,10-phenanthroline)dicyano: a Theoretical Study, Inorg. Chem., 2010, 49, 1634-1646.
41 W. Linert and V. Gutmann, Structural and electronic responses of coordination compounds to changes in the molecule and molecular environment, Coord. Chem. Rev., 1992, 117, 159-183.

42 A. Al-alousy and J. Burgess, Bis(1,10-phenanthroline) dicyanoiron(II): an almost universal inorganic solvent polarity indicator, Inorg. Chim. Acta, 1990, 169, 167-170.

43 L. Fodor, G. Lendvay and A. Horváth, Solvent Dependence of Absorption and Emission Spectra of Ru(bpy)2(CN)2: Experiment and Explanation Based on Electronic Structure Theory, J. Phys. Chem. A, 2007, 111, 12891-12900.

44 M. T. Indelli, C. A. Bignozzi, F. Scandola and J.-P. Collin, Design of Long-Lived Ru(II) Terpyridine MLCT States. Tricyano Terpyridine Complexes, Inorg. Chem., 1998, 37, 6084-6089.

45 J. R. Winkler, C. Creutz and N. Sutin, Solvent tuning of the excited-state properties of (2,2'-bipyridine)tetracyanoferrate(II): direct observation of a metal-to-ligand charge-transfer excited state of iron(II), J. Am. Chem. Soc., 1987, 109, 3470-3471.

46 T. J. Penfold, et al., X-ray Spectroscopic Study of Solvent Effects on the Ferrous and Ferric Hexacyanide Anions, J. Phys. Chem. A, 2014, 118, 9411-9418.

47 Note that while the $[\mathrm{Fe}(\mathrm{bpy})(\mathrm{CN}) 4]^{2-}$ is formally of $C_{2 \mathrm{v}}$ symmetry we refer to the $d-\pi$ and $d-\sigma^{*}$ orbitals with the octahedral eg and $\mathrm{t} 2 \mathrm{~g}$ notation to facilitate direct comparison between the different systems that are all near octahedral symmetry.

48 K. Haldrup, et al., Guest-Host Interactions Investigated by Time-Resolved X-ray Spectroscopies and Scattering at $\mathrm{MHz}$ Rates: Solvation Dynamics and Photoinduced Spin Transition in Aqueous Fe(bipy) ${ }_{3}{ }^{2+}$, J. Phys. Chem. A, 2012, 116, 9878-9887.

49 S. E. Canton, et al., Visualizing the non-equilibrium dynamics of photoinduced intramolecular electron transfer with femtosecond X-ray pulses, Nat. Commun., 2015, 6, 6359.

50 G. Vankó, et al., Detailed Characterization of a NanosecondLived Excited State: X-ray and Theoretical Investigation of the Quintet State in Photoexcited [Fe(terpy)2]2+ $2^{+}$. Phys. Chem. C, 2015, 119, 5888-5902.

51 Z. Fox, et al., Monitoring Excited State Charge Transfer of Transition Metal Mixed-Valence Complexes with Femtosecond X-ray Absorption and Emission Spectroscopy, International Conference on Ultrafast Phenomena (2016), UTh4A.2 (Optical Society of America, 2016), DOI: 10.1364/ UP.2016.UTh4A.2.

52 R. Alonso-Mori, et al., Photon-in photon-out hard X-ray spectroscopy at the Linac Coherent Light Source, J. Synchrotron Radiat., 2015, 22, 612-620.

53 M. Chollet, et al., The X-ray Pump-Probe instrument at the Linac Coherent Light Source, J. Synchrotron Radiat., 2015, 22, 503-507.

54 H. T. Philipp, M. W. Tate and S. M. Gruner, Low-flux measurements with Cornell's LCLS integrating pixel array detector, J. Instrum., 2011, 6, C11006.

55 R. Alonso-Mori, et al., A multi-crystal wavelength dispersive x-ray spectrometer, Rev. Sci. Instrum., 2012, 83, 073114. 
56 P. Glatzel and U. Bergmann, High resolution 1s core hole X-ray spectroscopy in $3 \mathrm{~d}$ transition metal complexes electronic and structural information, Coord. Chem. Rev., 2005, 249, 65-95.

57 H. E. Toma and M. S. Takasugi, Spectroscopic studies of preferential and asymmetric solvation in substituted cyanoiron(II) complexes, J. Solution Chem., 1983, 12, 547-561.

58 A. M. Brown, C. E. McCusker and J. K. McCusker, Spectroelectrochemical identification of charge-transfer excited states in transition metal-based polypyridyl complexes, Dalton Trans., 2014, 43, 17635-17646.

59 K. S. Kjær, et al., Ligand manipulation of charge transfer excited state relaxation and spin crossover in $\left[\mathrm{Fe}\left(2,2^{\prime}\right.\right.$ bipyridine)2(CN)2], Struct. Dyn., 2017, 4, 044030.

60 A. Horváth, J. Szõke and L. Wojnárovits, Mechanism of photoinduced redox reactions in aqueous solutions of [Fe(bpy)(CN)4]2- Inorg. Chim. Acta, 1991, 179, 97-104.

61 F. de Groot, High-Resolution X-ray Emission and X-ray Absorption Spectroscopy, Chem. Rev., 2001, 101, 1779-1808.
62 G. Vankó, et al., Probing the 3d Spin Momentum with X-ray Emission Spectroscopy: The Case of Molecular-Spin Transitions, J. Phys. Chem. B, 2006, 110, 11647-11653.

63 N. Lee, T. Petrenko, U. Bergmann, F. Neese and S. DeBeer, Probing Valence Orbital Composition with Iron $\mathrm{K} \beta$ X-ray Emission Spectroscopy, J. Am. Chem. Soc., 2010, 132, 9715-9727.

64 G. Vankó, et al., Spin-state studies with XES and RIXS: From static to ultrafast, J. Electron Spectrosc. Relat. Phenom., 2013, 188, 166-171.

65 N. A. Anderson, K. Hang, J. B. Asbury and T. Lian, Ultrafast mid-IR detection of the direct precursor to the presolvated electron following electron ejection from ferrocyanide, Chem. Phys. Lett., 2000, 329, 386-392.

66 M. Reinhard, et al., Photooxidation and photoaquation of iron hexacyanide in aqueous solution: A picosecond X-ray absorption study, Struct. Dyn., 2014, 1, 024901.

67 Q. Sun, et al., On the role of ligand-field states for the photophysical properties of ruthenium(II) polypyridyl complexes, Coord. Chem. Rev., 2015, 282-283, 87-99. 